\title{
Scatter Search and Path Relinking
}

\author{
Manuel Laguna \\ Leeds School of Business \\ University of Colorada \\ Boulder, CO 80309-0419 - USA \\ laguna@colorado.edu
}

This presentation explores the evolutionary approach called scatter search, which originated from strategies for creating composite decision rules and surrogate constraints. Recent studies have demonstrated the practical advantages of this approach for solving a diverse collection of optimization problems from both classical and real world settings. Scatter search contrasts with other evolutionary procedures, such as genetic algorithms, by providing unifying principles for joining solutions based on generalized path constructions in Euclidean space and by utilizing strategic designs where other approaches resort to randomization. Additional advantages are provided by intensification and diversification mechanisms that exploit adaptive memory, drawing on foundations that link scatter search to tabu search.

We also address the scatter search generalization called path relinking. Features that have been added to scatter search by extension of its basic philosophy, are captured in the path relinking framework. From a spatial orientation, the process of generating linear combinations of a set of reference solutions (as typically done in scatter search) may be characterized as generating paths between and beyond these solutions, where solutions on such paths also serve as sources for generating additional paths. This leads to a broader conception of the meaning of creating combinations of solutions. By natural extension, such combinations may be conceived to arise by generating paths between and beyond selected solutions in neighborhood space, rather than in Euclidean space.

The presentation also includes applications of scatter search/path relinking to multi-objective optimization and directions for future research. 